\title{
Création de référentiels commun : la coordination complexe du travail collaboratif
}

\section{Development of common repositories: the complex coordination of} collaborative work

Antoine Laurent ${ }^{1}$, Vanessa Lea ${ }^{1}$, Vincent Ard ${ }^{1}$, François Baleux ${ }^{1}$, Carine Calastrenc ${ }^{1}$, Ignacio Clemente Conte ${ }^{2}$, Daniel Condis ${ }^{3}$, Muriel Gandelin ${ }^{4}$, Ermengol Gassiot Ballbè ${ }^{5}$, Catherine Georjon $^{6}$, Florent Hautefeuille ${ }^{7}$, Mélanie Le Couédic ${ }^{8}$, Vivien Mathé ${ }^{9}$, Clément Moreau ${ }^{10}$, Joan Nunes Alonso ${ }^{5}$, Frédéric Pouget ${ }^{9}$, Christine Rendu ${ }^{11}$

${ }^{1}$ CNRS, UMR 5608 TRACES, antoine.laurent@univ-tlse2.fr

${ }^{2}$ IEEC - CSIC

${ }^{3}$ HADES

${ }^{4}$ CNRS, UMR 5608 TRACES

${ }^{5} \mathrm{UAB}$

${ }^{6}$ INRAP, UMR 5608 TRACES

${ }^{7}$ UT2J, MCF

${ }^{8}$ UPPA

${ }^{9}$ CNRS, UMR 7266 LIENSs, MCF

${ }^{10}$ ARCHEODUNUM, UMR 7264 CEPAM

${ }^{11}$ CNRS, UMR 5136 FRAMESPA

RÉSUMÉ. Les pratiques actuelles de la recherche en archéologie confirment une tendance forte au regroupement des chercheurs et à la mutualisation des données imposant désormais la mise en place de plateformes communes. Ce changement des pratiques se traduit par le passage du travail en local à l'ouverture vers des bases de données partagées entre plusieurs équipes, plusieurs institutions, voire plusieurs pays.

Depuis 2005, l'expérience de sept travaux réalisés au sein du laboratoire Traces (Architerre, ChasséoLab, Départ, Graph-Comp, Modelespace, Monumen, M\&P) en collaboration avec des institutions partenaires françaises et étrangères (ministère de la Culture, services régionaux, CNRS, universités, organismes et entreprises de l'archéologie préventive, associations, etc.) permet d'exposer les principes de la coordination, complexe, du travail collaboratif et l'impact des choix effectués.

ABSTRACT. Current archaeological research practices confirm a strong trend towards the grouping of researchers and the sharing of data, which now requires the establishment of shared platforms. This change in practices is reflected in the shift from working locally to opening up to databases shared by several teams, several institutions and even several countries.

Since 2005, the experience of seven projects carried out in the Traces laboratory (Architerre, ChasséoLab, Départ, Graph-Comp, Modelespace, Monumen, M\&P) in collaboration with French and foreign partner institutions (Ministry of Culture, regional services, CNRS, universities, preventive archaeology organizations and companies, associations, etc.) has helped to clarify the principles of coordination, complexity, collaborative work and the impact of the choices made.

MOTS-CLÉS. Archéologie, BD partagées, Webmapping, mutualisation, interopérabilité, gestion de projet.

KEYWORDS. Archaeology, online database, webmapping, sharing, interoperability, project management.

Lien vers la communication :

http://bit.ly/2RSgLfj 


\section{Introduction}

En archéologie, et plus largement dans le monde des sciences humaines et sociales, les nouvelles technologies numériques révolutionnent la recherche en équipe autour de projets fédérateurs nécessitant la mise en place de règles pour la gestion et la coordination du travail collaboratif. Le développement des outils informatiques et leur accès en ligne incitent notamment de plus en plus les chercheurs à mutualiser les données dans des référentiels communs. A partir de sept projets conduits depuis 2007 au sein du laboratoire TRACES (UMR 5608, Université Toulouse Jean Jaurès), cette présentation entend aborder cette thématique transversale de manière plus méthodologique que technique.

Les projets couvrent une large emprise chronologique, depuis le Néolithique jusqu'à l'époque contemporaine, et conçoivent la base de données partagées soit comme un point de départ à une recherche, soit comme un objectif final de rendu.

Par ordre alphabétique :

- ANR Architerre : l'objectif général est de mettre en place une interface web susceptible d'intégrer toutes les sources écrites médiévales et modernes liées à la fiscalité de la terre permettant d'étudier la structuration des paysages.

- GDR ChasséoLab : la mission de ce Groupement De Recherche est double : il s'agit d'une part d'effectuer un bilan sur le Chasséen méridional (inventaire des sites, mobiliers et données environnementales) et d'autre part de structurer la recherche à venir à la lumière de ce bilan. Le Chasséen est en effet loin de faire consensus quant à sa définition, la délimitation de son expansion géographique, ses bornes chronologiques ou encore sa périodisation interne.

- Groupement Départ : le réseau DEPART étudie les Dynamiques des Espaces Pyrénéens d'Altitude grâce à un Système d'Information Géographique sur le patrimoine archéologique et la modélisation des territoires pastoraux en France, en Andorre et en Espagne.

- ANR Graph-comp : par une approche interdisciplinaire, ce projet a pour objectif d'étudier les réseaux sociaux au sein du monde paysan à travers le prisme d'un étroit secteur géographique de 3 communes du département du Lot. Pour cela il utilise la méthode des graphes appliquée à la documentation historique de la fiscalité de la terre médiévale et moderne.

- ANR Modelespace : l'ambition de cette recherche est d'analyser les dynamiques spatiales des peuplements à partir de sources fiscales sérielles de natures différentes. L'outil développé permet d'intégrer aux processus d'analyses déjà bien maîtrisés à partir de représentations cartographiques de l'espace (plans cadastraux, photographies aériennes...) des documents dépourvus de plan, tels que les sources fiscales médiévales et modernes.

- ANR MONUMEN a pour objectif d'analyser l'émergence et le développement des plus anciennes expressions du monumentalisme architectural en Europe occidentale, qui émergent au Néolithique au milieu du $5^{\mathrm{e}}$ millénaire avant notre ère, à la fois dans le monde des morts (mégalithes) et dans le monde des vivants (enceintes). L'outil de WebSIG a pour objectif d'interroger et de cartographier les données acquises sur les différentes fenêtres du projet, entre Loire et Pyrénées.

- PCR Milieu et Peuplement est un Projet Collectif de Recherche qui étudie le Néolithique au Bronze ancien en Languedoc occidental.

\section{Le travail connecté en équipe : de nouvelles pratiques}

La recherche actuelle est basée sur des projets ayant des ambitions élevées et des modes de financements divers (GDR, ANR, PCR). Cela a pour effet une inflation du nombre des acteurs au sein 
d'équipes pluridisciplinaires parfois nouvellement constituées. Les projets regroupent de vingt à une centaine de spécialistes provenant d'organismes différents : Ministère de la Culture, services régionaux de l'archéologie, CNRS, universités, organismes et entreprises de l'archéologie préventive, associations... A cette pluralité d'institutions s'ajoutent des échelles spatiales d'analyses variées, allant de focus locaux jusqu'à l'échelle de la France, tout en franchissant régulièrement les frontières nationales.

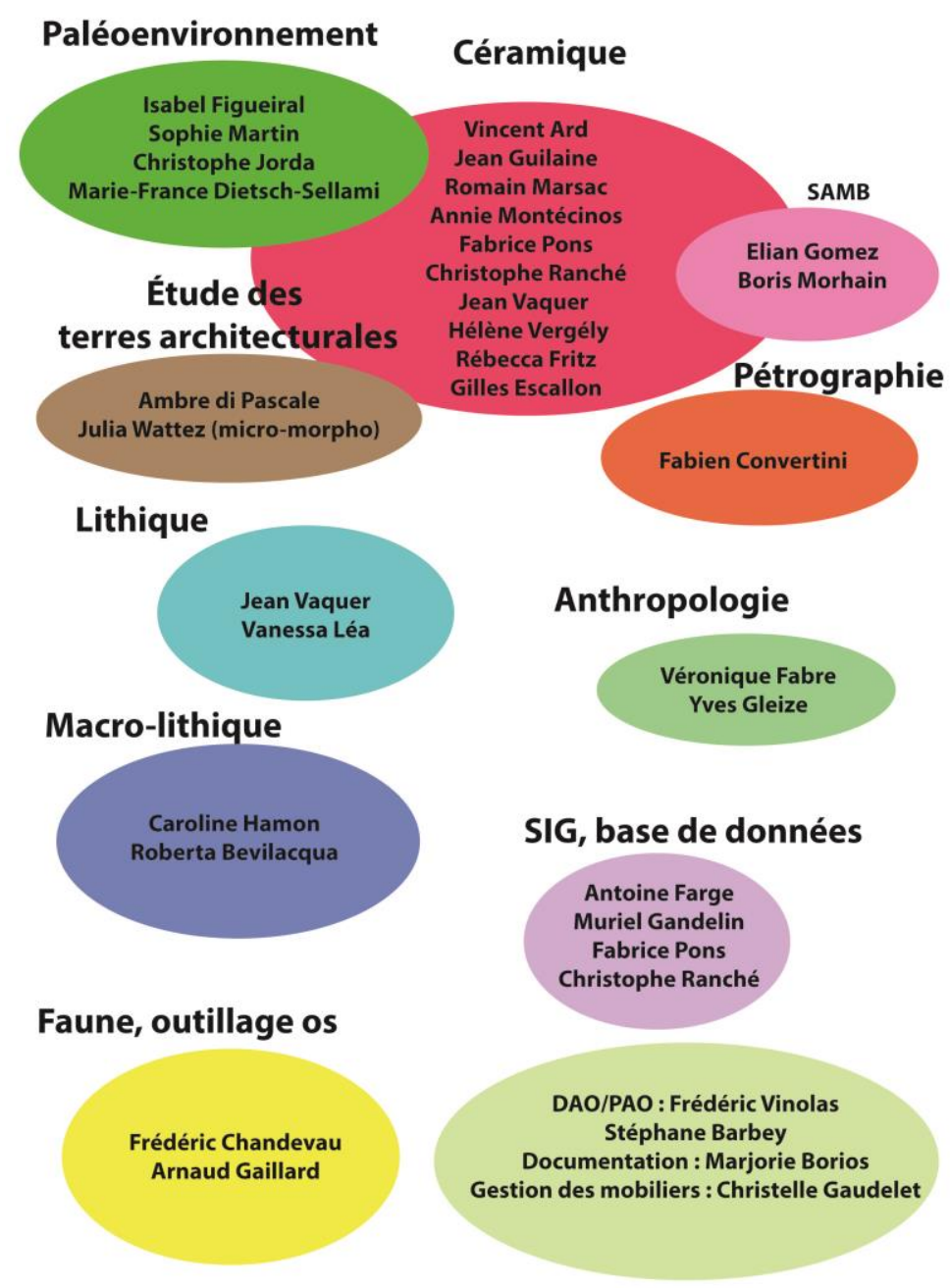

Figure 1. M\&P, ensemble des spécialités.

Ces équipes pluridisciplinaires multiplient les types de données numériques créées qu'il faut mettre en commun. A chaque nouveau programme, les chercheurs font face à un accroissement du nombre de sites étudiés passant de quelques dizaines à plusieurs centaines, voire milliers d'entités. Les bases de données et les outils d'analyses deviennent alors des dispositifs indispensables. Le Système d'Information Géographique (SIG) est régulièrement employé pour sa capacité à traiter l'information spatialisée. Il centralise et croise des sources de nature variée et permettent l'analyse et la modélisation de phénomènes.

Pour répondre à ces défis, les programmes de recherche se tournent vers les bases de données en ligne et/ou partagées. 


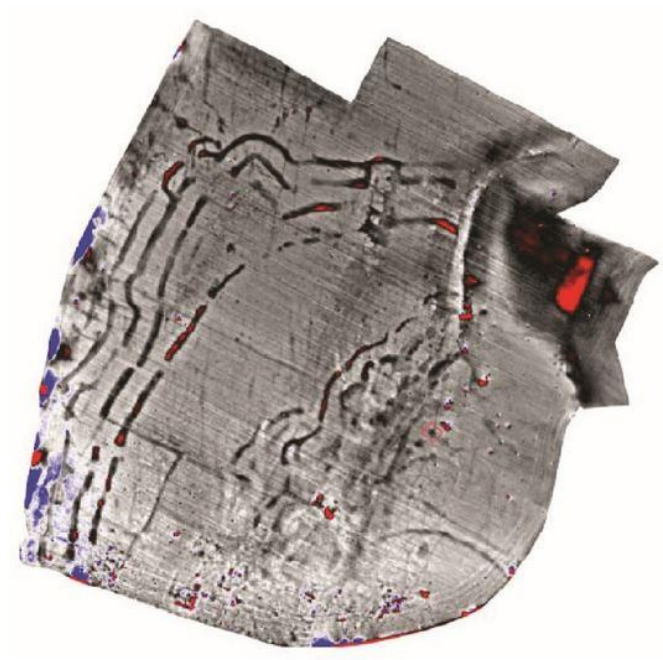

Enceintes fossoyées

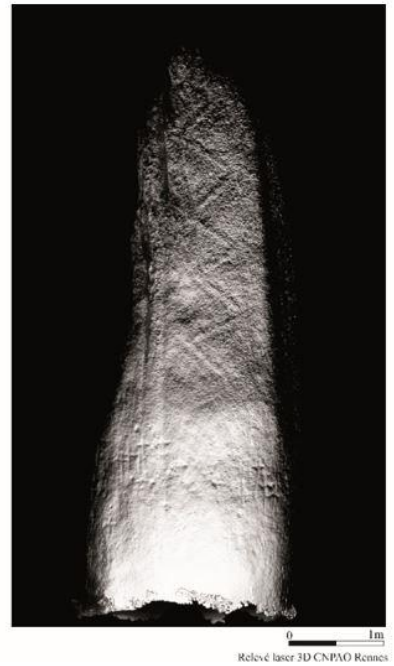

Stèles gravées

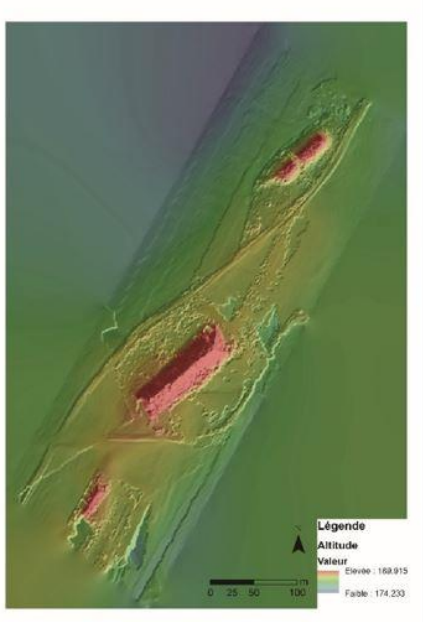

Tumulus

Figure 2. MONUMEN, l'étude des différentes expressions du mégalithisme.

\section{Comité de pilotage}

La plupart des projets étudiés dans cet article sont composés d'axes thématiques avec en rôle centralisateur accordé à la construction des outils de gestion de la donnée. Dans le cadre de ChasséoLab un comité de pilotage à plusieurs niveaux supervise l'élaboration du référentiel commun. Un Conseil examine le bilan effectué annuellement par les coordinateurs d'ateliers. Ce conseil est composé de sept personnes de différentes institutions et spécialités dans un souci du respect de la représentativité de ces dernières. Les membres du Conseil ne sont pas directement impliqués scientifiquement dans le GDR. Treize coordinateurs sont répartis dans chaque atelier qu'ils animent par équipe de trois à cinq. Ils sont responsables du travail scientifique effectué au sein des ateliers. Certaines réunions associent les coordinateurs par atelier (programmation du travail pour l'année, rédaction des comptes-rendus, debriefing après une séance...) ; d'autres regroupent la totalité des coordinateurs (planification du travail de la totalité du GDR à l'année, difficultés rencontrées, distribution des crédits et gestion du budget annuel, choix stratégiques...).
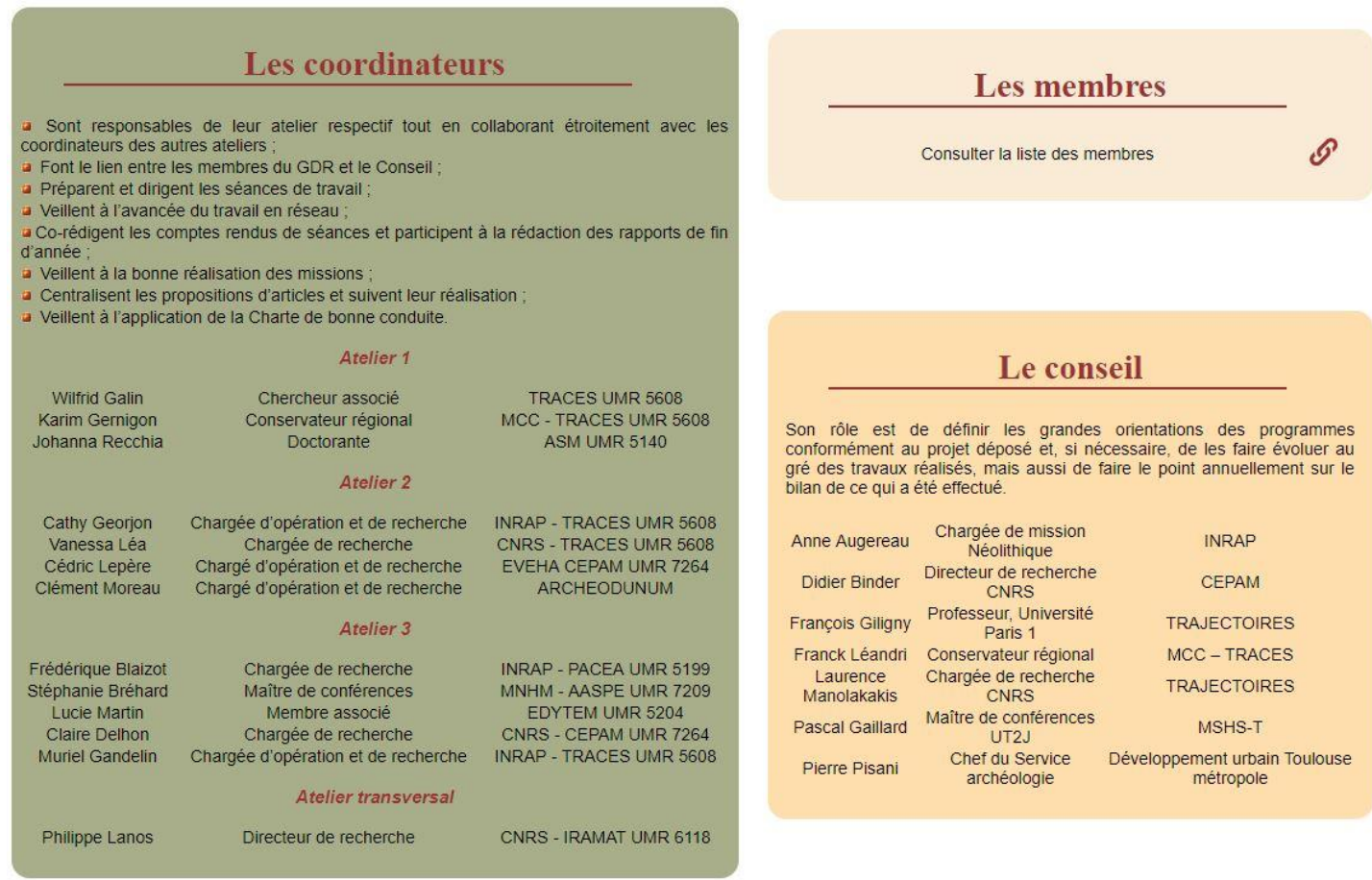

Figure 3. ChasséoLab, la composition du comité de pilotage. 
La liste des institutions et des partenaires est souvent longue avec une pluralité des statuts et des profils des chercheurs ; cela demande l'élaboration d'un cadre normatif. Pour le projet ChasséoLab, la mise en place du cadre déontologique a nécessité deux ans de travail aboutissant à un Accord de Consortium qui règle les problèmes de propriété intellectuelle. Il est signé par tous les partenaires publiques et privés : les données émanant d'un partenaire restent la propriété de ce partenaire ; celles élaborées collégialement dans le cadre du GDR sont la propriété du GDR. Les signatures des articles sont de même réglementées, tout comme les références à des rapports, des données détenues par d'autres, etc. Cet accord est traduit par une Charte de bonne conduite à destination de tous les membres du GDR. Il prévoit de même une convention d'accueil pour les salariés du privé : par sa signature, les entreprises partenaires s'engagent à garantir un nombre de jours/homme par an et à prendre en charge les frais de missions des salariés participant au projet. La fiche, nominale, est signée par l'une des porteuses du projet (Vanessa Lea), la société privée et les salariés concernés. Ces derniers sont donc soumis aux même règles de bonne conduite et au même respect de l'Accord de Consortium que les partenaires publics.

De plus, les projets français d'archéologie prennent en compte la protection et de la sensibilité des données de la base Patriarche. Toutes les questions d'échanges avec les services des DRAC ou des SRA sont à intégrer dans la coordination des acteurs.

\section{Définition du référentiel commun}

Qu'il s'agisse d'élaborer un projet à partir de données anciennes pour tendre vers une nouvelle typologie ou bien de créer de nouvelles sources de données (fouilles, prospections, données environnementales, technologies 3D...) la définition du «commun» est déterminante dans le déroulement des travaux de recherche. Comment intégrer, adapter, lier ou reprendre des bases de données ou des sources anciennes? Comment sont gérées des données collectées avec des méthodologies variées et comportant bien souvent des hiatus?

Les réponses se construisent au cours des réunions et des workshops thématiques. Dans le cadre du Réseau Départ qui s'intéresse aux structures pastorales des deux côtés des Pyrénées, les intervenants élaborent les définitions du référentiel en partant d'un mot ou d'une photographie d'un site. De là, chaque caractéristique est étudiée et catégorisée avec ses paramètres propres. Ces moments d'échange sont révélateurs des différences que peuvent couvrir certaines notions. L'une des difficultés rencontrées a été la mise en exergue des différences de sens attribuées aux mots désignant un même objet qui n'a pu être réglé que par la création d'une base de données multilingue s'appuyant sur un thésaurus traduit (en français, espagnol, basque et catalan). Ainsi les membres du projet utilisent la base dans sa propre langue que ce soit lors de la saisie ou de l'interrogation des données. 


\section{Croisement}

\begin{tabular}{|c|c|c|c|c|c|c|c|c|c|c|c|}
\hline \multirow[b]{3}{*}{ Fonction1 } & \multicolumn{11}{|c|}{ Type de Structure } \\
\hline & \multicolumn{4}{|c|}{ Structure Architectonique } & \multicolumn{4}{|c|}{ Cavité Naturelle } & \multicolumn{3}{|c|}{ Espace Ouvert } \\
\hline & Cabane & Endos & Mur & Indeterminée & Grote & Bame & Abri-sous-roche & Indeterminée & Mobiliere & Niveau de sol & Indeterminée \\
\hline \multirow[t]{2}{*}{ Habiat } & Habiation & & & & Habiation & Habiation & & & & Habiafion & \\
\hline & Revige & & & & Revige & Revige & Retige & & & Revige & \\
\hline \multirow[t]{4}{*}{ Parcage } & Abri à agneaux & Corral ?? & & & Bergerie?? & Bergerie?? & Abri à agneaux & & & Corral ?? & \\
\hline & Niche à chien & & & & & & Niche à chien & & & Bergerie?? & \\
\hline & & & & & & & & & & Abri à agneaux & \\
\hline & & & & & & & & & & Niche à chien & \\
\hline Habitat:Parcage & & & & & $? ?$ & $? ?$ & & & & $? ?$ & \\
\hline Parcage/Production & & Couloir a trate & & & & & & & & & \\
\hline \multirow[t]{3}{*}{ Stockage } & Magasin & & & & Magasin & Magasin & Magasin & & & Magasin & \\
\hline & Cave à fromage & & & & Cave à tromage & Cave à tromage & Cave à fromage & & & Cave à tromage & \\
\hline & & & & & Cache & Cache & Cache & & & Cache & \\
\hline \multirow[t]{2}{*}{ Production/Transiormation } & Atelier & & & & Atelier & Atelier & Atelier & & Ateler & Ateler & \\
\hline & Saloir & & & & Saloir & Saloir & Saloir & & & Saloir & \\
\hline Triage/Comptage & & 'Pas compiador' & 'Pas complador' & & & & & & & & \\
\hline Indeterminée & Indeterminée & Indeterminée & Indeterminée & Indeterminée & Indeterminee & Indeterminée & Indeterminée & Indeterminée & Indeterminée & Indeterminèe & Indeterminé \\
\hline
\end{tabular}

Figure 4. Réseau Départ, tableau du croisement des données.

Pour le cas de ChasséoLab l'ambition se porte sur de nouvelles définitions des caractéristiques du Chasséen. Tout un pan du travail concerne l'emploi par chaque spécialiste d'un vocabulaire, d'une typologie ou d'une terminologie propre à un espace particulier. Dans un esprit de synthèse, des vignettes-dessins représentant les vases permettent de dépasser l'utilisation du vocabulaire controversé pour atteindre un consensus. C'est à partir de ce travail effectué collégialement que sera établi un glossaire commun.

Profil général

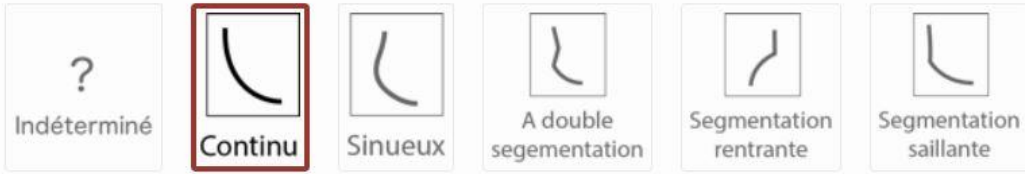

Morphologie panse

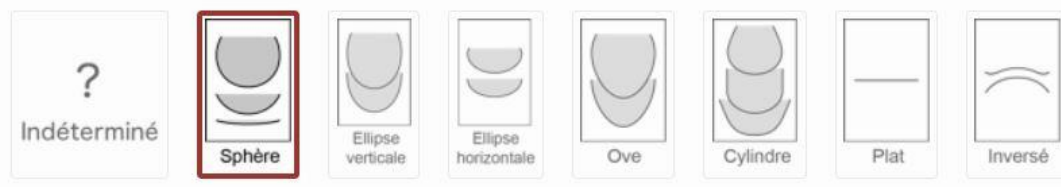

Type de lèvre, bord et rebord particulier

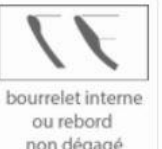

non dégage

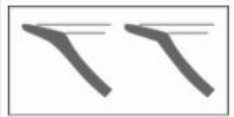

rebord dégagé

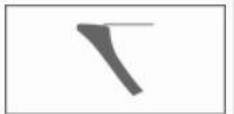

rebord court épais

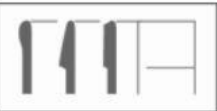

bord « renforcé »

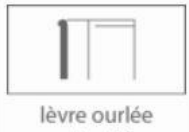

vers l'extérieur

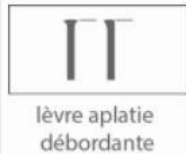

Figure 5. ChasséoLab, obtenir un consensus avec des vignettes-dessins.

Une autre difficulté rencontrée concerne l'unité d'enregistrement: quel est le niveau adapté aux problématiques ? est-il suffisant en terme de résolution et/ou de précision ? 


\section{Structuration de la base de données}

Les questionnements sur le référentiel commun aboutissent là des choix au niveau de la structuration et du format de la base, ainsi que des outils à y implémenter. Ces décisions primordiales peuvent influencer la conception du référentiel par les capacités fonctionnelles des logiciels ou encore par l'expertise nécessaire pour les mettre en œuvre. Du dictionnaire des champs au Modèle Conceptuel de Données se joue la complexité de la future base avec un nombre variable de tables. Grâce au fonctionnement des projets sur plusieurs années et avec des financements assurés, le recours aux contractuels est rendu possible. Ils apportent une forte spécialisation et des possibilités de développement plus importantes mais posent la question d'un suivi technique parfois en décalage avec les rythmes de la recherche.

Début de réflexions sur la base de données relationnelle Inicio de una reflexión sobre la base de datos relational

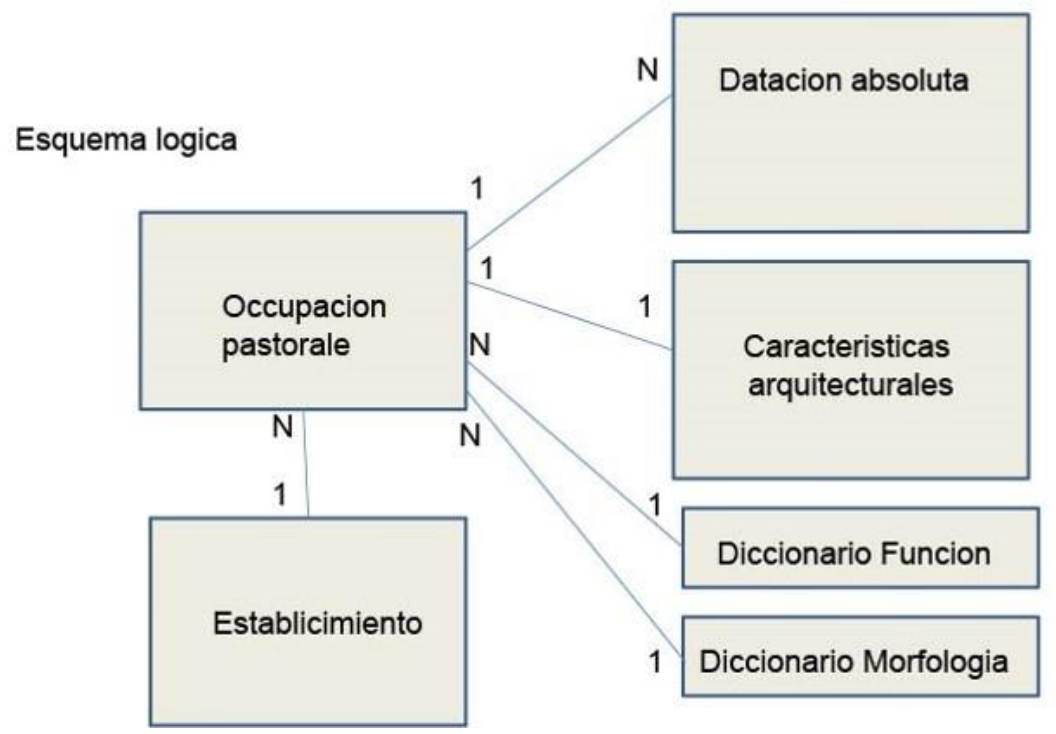

Figure 6. Réseau Départ, le choix d'un MCD avec une conception simple.

Dans ce corpus d'étude, les bases de donnée sont des Systèmes de Gestion de Bases de Données Relationnelles (SGBDR) en SQL (PostgreSQL, MySQL, Access ou FileMaker). L'hébergement se tourne de plus en plus vers la TGIR Huma-Num plutôt que sur des serveurs universitaires.

\section{Connexion des outils}

A partir de la base de données se connectent les interfaces de saisie et de visualisation. Les formulaires, de manière générale, se présentent de la même façon et suivent les thématiques du projet. Pour une harmonisation de la donnée enregistrée, les listes déroulantes et les cases à cocher sont favorisées, en limitant le plus souvent la saisie libre aux champs de commentaire. 


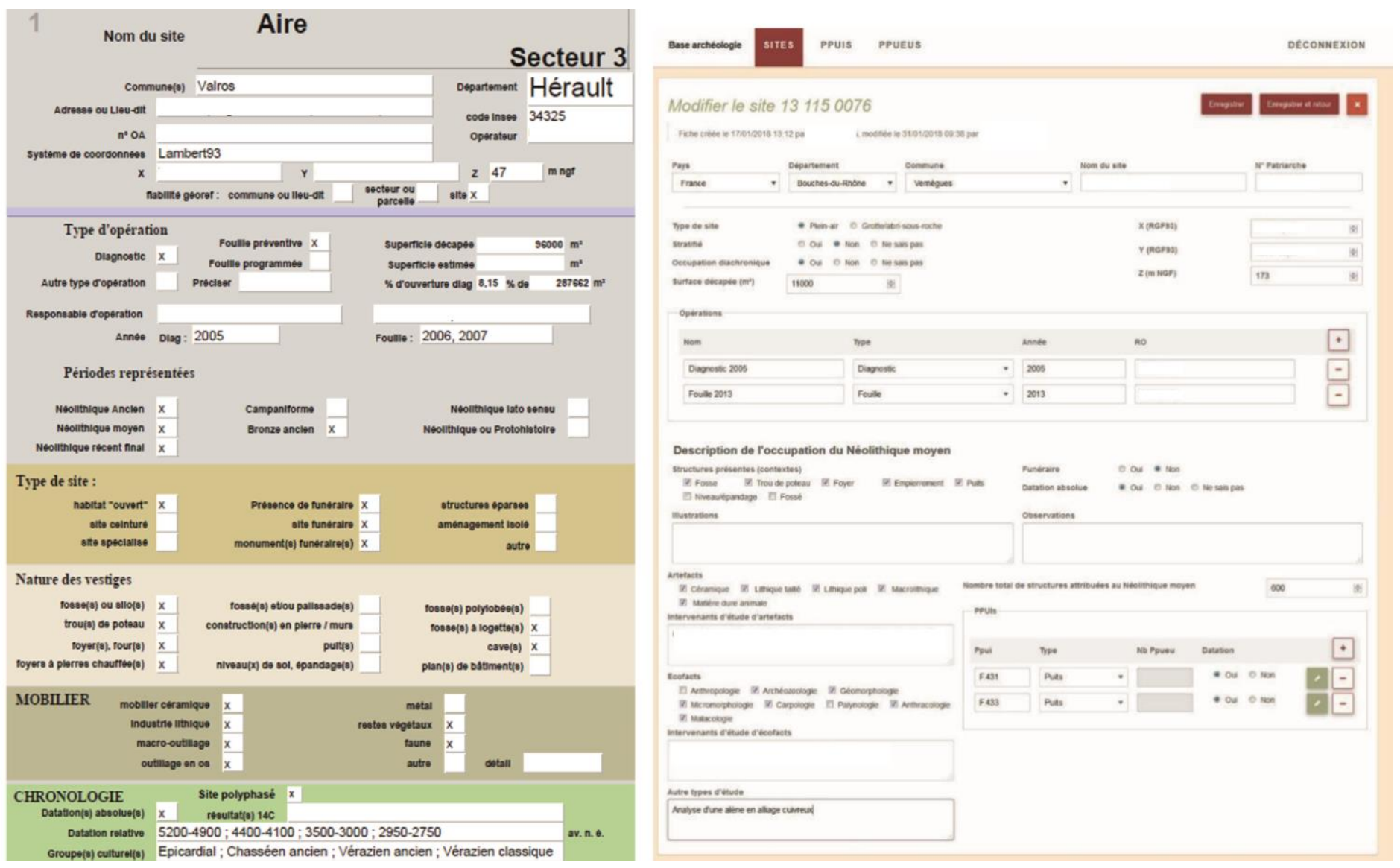

Figure 7. M\&P et ChasséoLab, masque de saisie.

Les interfaces en ligne, selon les technologies utilisées, permettent de développer des fonctionnalités particulières et adaptées aux besoins des chercheurs. Dans le cadre du PCR Sel inclus dans l'ANR Monumen, des champs automatiques de la fiche information permettent de récupérer les coordonnées et l'altitude des entités ajoutées. Une originalité de la saisie des objets lithique du projet ChasséoLab est la visualisation en temps réel, graphique et dynamique des proportions des objets.

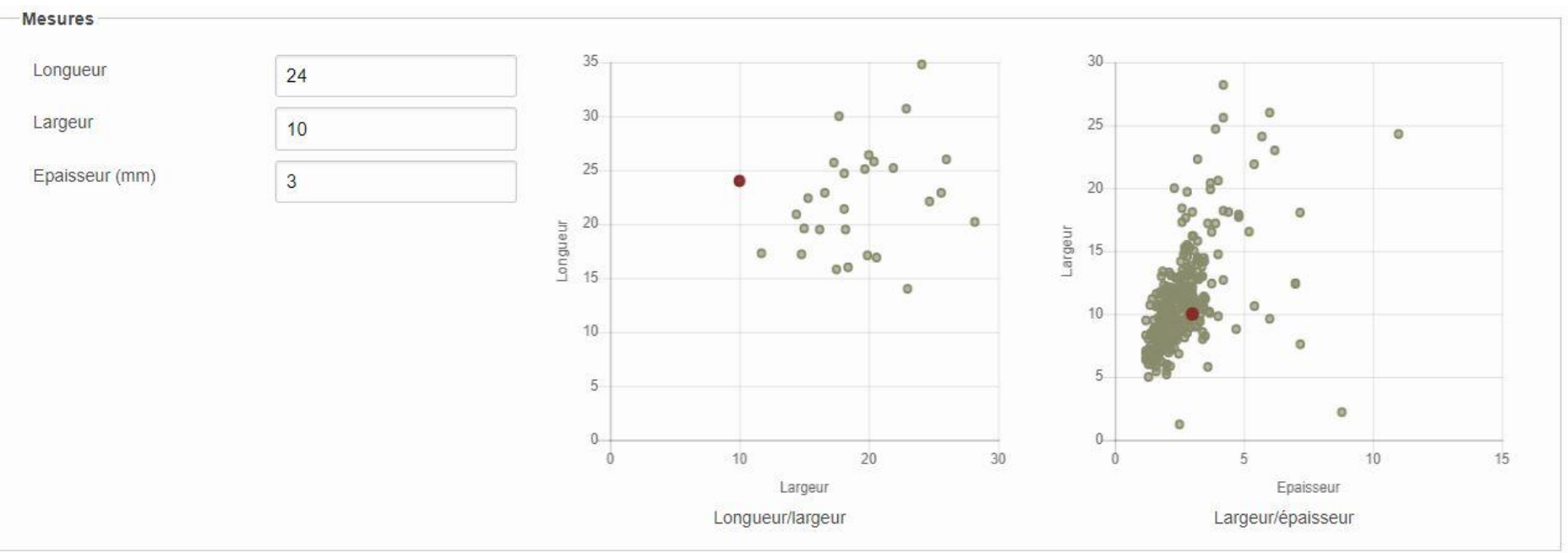

Figure 8. ChasséoLab, mesure des objets lithique.

Selon le choix d'ouvrir la base à un public élargi ou non, toutes les interfaces intègrent des modules de requêtes. Certaines permettent de simple filtre sur des champs prédéfinis ou alors des requêtes multicritères et multivaluées construites par l'utilisateur. L'objectif est autant d'accéder à l'information que de pouvoir l'exporter. 
2 Choix des critères

\begin{tabular}{|c|c|c|c|c|c|c|}
\hline \multirow{2}{*}{$\begin{array}{l}\text { + Ajouter un critère } \\
\text { Datations }\end{array}$} & \multirow[b]{2}{*}{$\checkmark$} & \multicolumn{4}{|c|}{ - Correspondance à tous les critères (ET) } & \multirow{2}{*}{$\begin{array}{l}\text { Correspondance à au moins un critère }(\mathrm{OU}) \\
6000\end{array}$} \\
\hline & & Age BP & $\checkmark$ & plus petit que & $\checkmark$ & \\
\hline Datations & $\checkmark$ & Age BP & 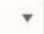 & plus grand que & $\checkmark$ & 5000 \\
\hline
\end{tabular}

(3) Choix des champs à afficher

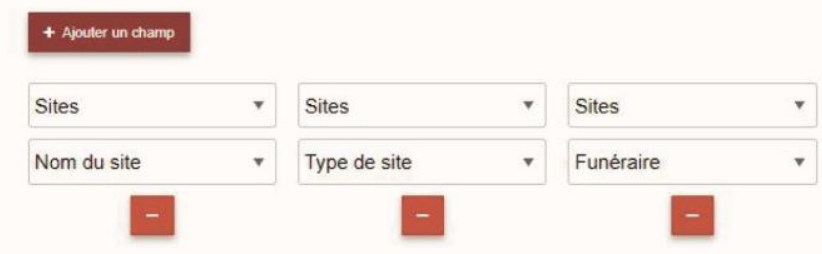

\begin{tabular}{|l|l|l|l|l|}
\hline \#Afficher les résultats & व Générer un fichier & \#Planche images & OVoir dans Géo & MEnregistrer la requête \\
\hline
\end{tabular}

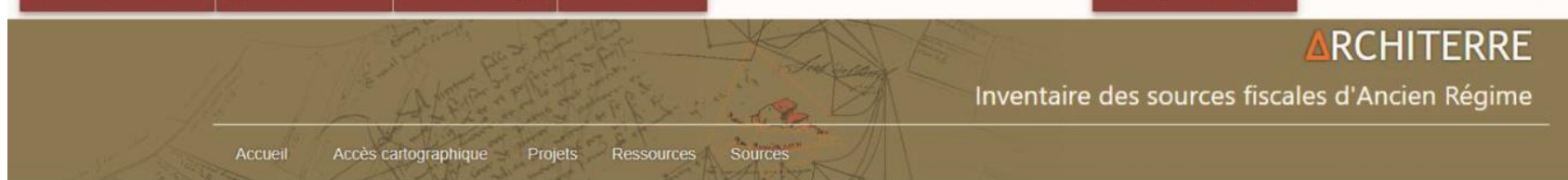

Recherche de ressources

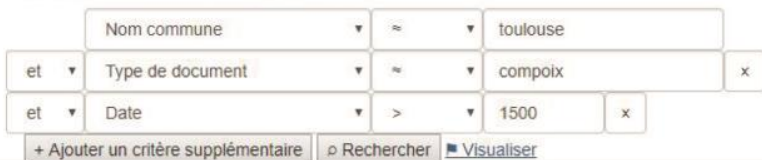

\begin{tabular}{|c|c|c|c|c|c|c|c|c|}
\hline Nom commune & Type & Désignation d'inventaire & Date & Complément date & Service de conservation & Lieu de conservation & Côte & Description \\
\hline TOULOUSE & compoix & cadastre & 1550 & & Arch. Mun. Toulouse & Toulouse & CC 20 à 34 & CC $20=$ Cadastr.. \\
\hline TOULOUSE & compoix & arpentage & 1571 & & Arch. Mun. Toulouse & Toulouse & CC 35 a cC 48 & CC $35=1571,2 \ldots$ \\
\hline TOULOUSE & compoix & cadastre & 1669 & & Arch. Mun. Toulouse & Toulouse & CC 57 à 68 & CADASTRE $d u X$... \\
\hline TOULOUSE & compoix & & 1679 & & Arch. Mun. Toulouse & Toulouse & $\operatorname{CC} 69$ à 75 & CADASTRE du $X$ \\
\hline TOULOUSE & compoix & matrice & 1681 & & Arch. Mun. Toulouse & Toulouse & CC 76 à 113 & CADASTRE de $1 \ldots$ \\
\hline IEILLE-TOULOUSE & compoix & compoix & 1545 & & Arch Mun. Vieille-Toulouse & Vieille-Toulouse & 1 NUM AC 3624. & Commune de Vie... \\
\hline
\end{tabular}

Figure 9. ModelEspace et ChasséoLab, module de requête.

La cartographie a toujours eu une place importante dans les travaux archéologiques, mais son niveau d'expertise en limitait son utilisation. Le développement des outils de Webmapping (cartographie en ligne) et de WebSIG (édition attributaire et géométrique en ligne) offrent de nouvelles perspectives d'accès et de visualisation de la donnée. Ils peuvent s'entrevoir comme une plateforme indépendante ou comme l'affichage d'un résultat de requête. 


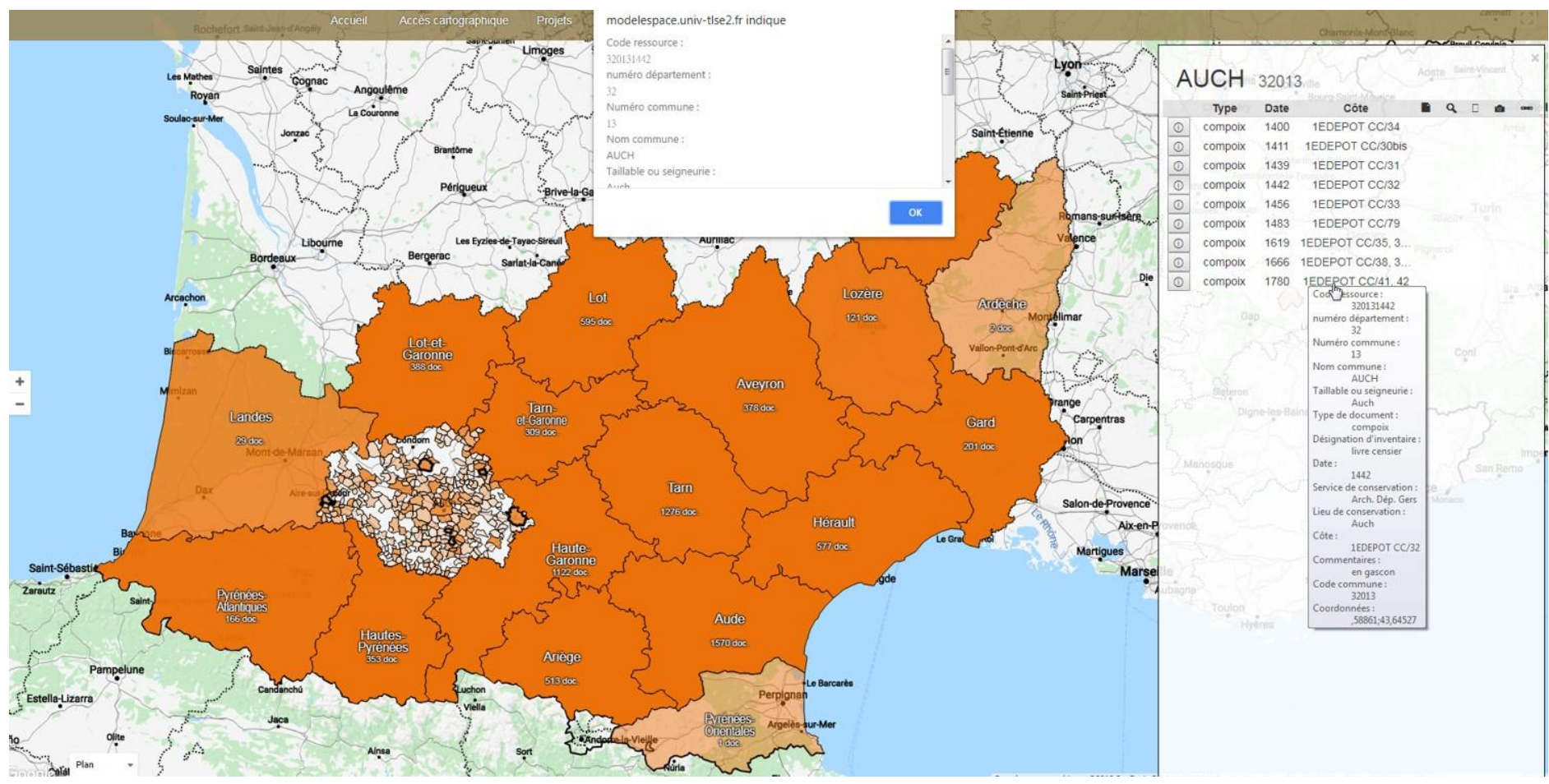

Figure 10. Architerre, interface cartographique publique.

L'analyse de réseau par la génération de graphe est encore marginale dans les projets archéologiques. Provenant des sciences sociales, cet méthode d'analyse permet dans le cadre du projet Graph-Comp de dépasser les limites du SIG et de faire abstraction du réel géographique. A partir de sources fiscales médiévales, qu'elles soient écrites (compoix, terriers) ou graphiques (plans et cartes), des relations sont établies entre des hommes et des parcelles aboutissant à la reconstitution des paysages médiévaux et de leurs évolutions.
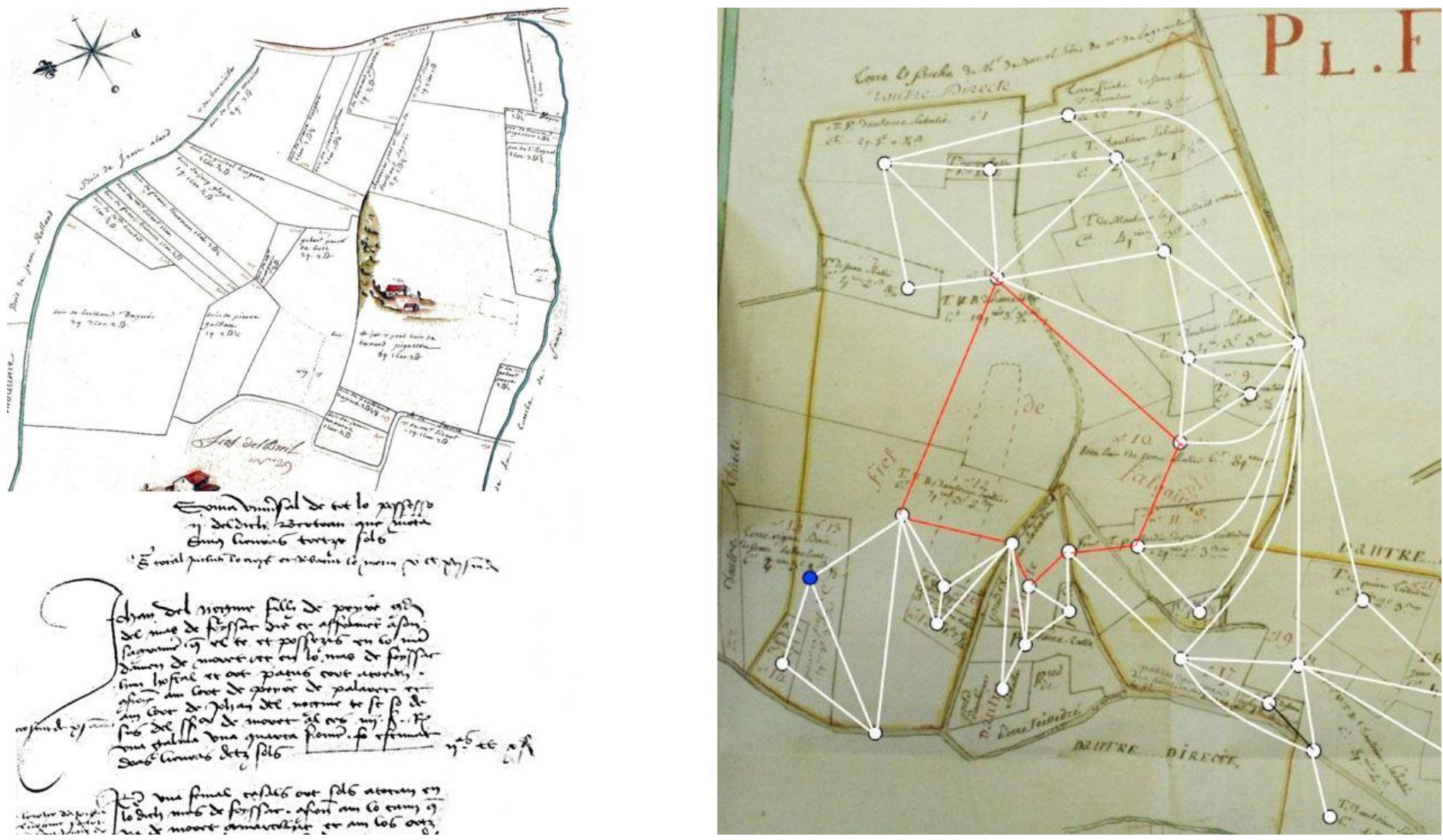

Figure 11. Graph-Comp, visualisation de graphe. 
L'une des difficultés de l'analyse de réseau médiéval est la complexité du développement de l'outil. Pour des raisons diverses, la construction du graphe est à opérer par l'utilisateur. Cela adjoint une étape supplémentaire qui s'ajoute à la difficulté de compréhension des réseaux.

Les outils ont une part importante dans le succès de la constitution d'un référentiel commun. C'est au comité de pilotage de bien recenser les besoins et les fonctionnalités nécessaires au projet. La clé se situe dans la capacité de communication entre les maîtres d'œuvres et les maîtres d'ouvrages.

\section{Conclusion}

Chaque projet est unique dans ses démarches scientifiques et son développement technique. Toutefois, tous les choix tendent vers l'interopérabilité entre les outils et vers une meilleure diffusion de l'information entre les partenaires. La notion de FAIR data (Findable, Accessible, Interoperable, Reusable) et la rédaction plus régulière de Data Management Plan (DMP) attestent ce constat.

Toutefois des pratiques ralentissent encore la diffusion de l'information entre projets :

- Création d'une structure de données à chaque nouveau programme de recherche,

- Manque d'accès aux métadonnées et aux protocoles de création de la donnée (thésaurus),

- Utilisation de formats et d'encodages pas toujours standardisés,

- Maintien des liens dynamiques et pérennes entre les bases,

- Maintien des interfaces après la durée des projets.

Lors de la création de référentiels communs il est primordial que l'équipe coordinatrice prenne en compte la compatibilité de la base de données et de l'information publiée avec des banques centralisatrices telles que Patriarche, Ariadne, Isidore, Arkéogis, CArGOS ou encore Péripléo.

Plus largement, le travail collaboratif en ligne est à penser comme une nouvelle archive numérique et sa pérennité doit être envisagée et garantie dès l'amont du projet.

\section{Bibliographie et Webographie (dernière consultation le 12/02/19) :}

ABBE, J-L., HAUTEFEUILLE, F. (dir.) 2017. Estimes, compoix et cadastres. Histoire d'un patrimoine commun de l'Europe méridionale, Toulouse, Le Pas d'oiseau, 271 p.

HAUTEFEUILLE, F. 2016. "Géolocalisation des sources fiscales pré-révolutionnaires : la quadrature du cercle », Bulletin du centre d'études médiévales d'Auxerre | BUCEMA, Hors-série $\mathrm{n}^{\circ} 9$ (http://journals.openedition.org/cem/13800)

LE COUEDIC, M., RENDU, C., GARCIA CASAS, D., GASSIOT BALLBE, E., CALASTRENC, C. et al.. Comparer et modéliser les sites, les territoires et les systèmes pastoraux pyrénéens dans la diachronie : présentation et premiers résultats du projet collaboratif DEPART. ,dans DEBOFLE, P., SANCHEz, J-C., Pays Pyrénéens et environnement, Juin 2015, Bagnères-de-Bigorre, France. pp.321-338, 2016, Pays pyrénéens et environnement. Actes du 62e Congrès de la Fédération historique de Midi-Pyrénées, Bagnères-de-Bigorre (Hautes-Pyrénées), 12-13-14 juin 2015 : 150e anniversaire de la Société Ramond (1865-2015) dans le cadre des commémorations nationales 2015. (〈halshs01399071) ).

\section{Projet de l'UMR 5608 TRACES :}

- http://modelespace.univ-tlse2.fr - Site des projets ANR ModelEscpace et Architerre

- https://chasseolab.huma-num.fr - Site du GDR ChasséoLab

- $\quad$ http://graphcomp.univ-tlse2.fr - Site de l'ANR Graph-Comp

- http://monumen.huma-num.fr - Site de l'ANR Monumen 
Service et outil en ligne :

- https://www.huma-num.fr - Site de la TGIR des humanités numériques

- https://isidore.science/ - Assistant de recherche en SHS

- https://dmp.opidor.fr - Réalisation de DMP en ligne sur DMP OPIDoR

- https://ariadne-infrastructure.eu - Consortium Ariadne

- http://arkeogis.org/ - Plateforme ArkeoGIS

- https://peripleo.pelagios.org/ - Plateforme Peripleo

- http://cargos.huma-num.fr/ - Catalogue de données géographiques en SHS 